University of Nebraska - Lincoln

DigitalCommons@University of Nebraska - Lincoln

Faculty Publications in the Biological Sciences

Papers in the Biological Sciences

$4-6-2001$

\title{
Extension of Life-Span by Loss of CHICO, a Drosophila Insulin Receptor Substrate Protein
}

\author{
David Clancy \\ University College London, Wolfson House, 4 Stephenson Way, London \\ David Gems \\ University College London, Wolfson House, 4 Stephenson Way, London \\ Lawrence G. Harshman \\ University of Nebraska - Lincoln, Iharshman1@unl.edu \\ Sean Oldham \\ Universität Zürich, Winterthurerstrasse 190, 8057 Zürich, Switzerland \\ Hugo Stocker \\ Universität Zürich, Winterthurerstrasse 190, 8057 Zürich, Switzerland \\ See next page for additional authors
}

Follow this and additional works at: https://digitalcommons.unl.edu/bioscifacpub

Part of the Life Sciences Commons

Clancy, David; Gems, David; Harshman, Lawrence G.; Oldham, Sean; Stocker, Hugo; Hafen, Ernest; Leevers, Sally; and Partridge, Linda, "Extension of Life-Span by Loss of CHICO, a Drosophila Insulin Receptor Substrate Protein" (2001). Faculty Publications in the Biological Sciences. 9.

https://digitalcommons.unl.edu/bioscifacpub/9

This Article is brought to you for free and open access by the Papers in the Biological Sciences at DigitalCommons@University of Nebraska - Lincoln. It has been accepted for inclusion in Faculty Publications in the Biological Sciences by an authorized administrator of DigitalCommons@University of Nebraska - Lincoln. 


\section{Authors}

David Clancy, David Gems, Lawrence G. Harshman, Sean Oldham, Hugo Stocker, Ernest Hafen, Sally Leevers, and Linda Partridge 
Published in Science 292 (April 6, 2001), pp. 104-106; doi 10.1126/science.1057991 Copyright () 2001 American Association for the Advancement of Science. Used by permission. http://www.sciencemag.org/cgi/content/full/292/5514/104

Submitted December 4, 2000; accepted March 7, 2001.

\title{
Extension of Life-Span by Loss of CHICO, a Drosophila Insulin Receptor Substrate Protein
}

\author{
David J. Clancy, ${ }^{1}$ David Gems, ${ }^{1}$ Lawrence G. Harshman, ${ }^{2}$ Sean Oldham, ${ }^{3}$ \\ Hugo Stocker, ${ }^{3}$ Ernst Hafen, ${ }^{3}$ Sally J. Leevers, ${ }^{4,5}$ and Linda Partridge ${ }^{1}$
}

\section{Department of Biology, University College London, Wolfson House, 4 Stephenson Way, London NW1 2HE, UK. \\ 2 School of Biological Sciences, University of Nebraska-Lincoln, Lincoln, NE 68588, USA. 3 Zoologisches Institut, Universität Zürich, Winterthurerstrasse 190, 8057 Zürich, Switzerland. 4 Ludwig Institute for Cancer Research, 91 Riding House Street, London W1W 7BS, UK. \\ 5 Department of Biochemistry and Molecular Biology, University College London, Gower Street, London WC1E 6BT, UK. \\ Corresponding author - D. Gems, email david.gems@ucl.ac.uk}

\begin{abstract}
The Drosophila melanogaster gene chico encodes an insulin receptor substrate that functions in an insulin/insulin-like growth factor (IGF) signaling pathway. In the nematode Caenorhabditis elegans, insulin/IGF signaling regulates adult longevity. We found that mutation of chico extends fruit fly median life-span by up to $48 \%$ in homozygotes and $36 \%$ in heterozygotes. Extension of life-span was not a result of impaired oogenesis in chico females, nor was it consistently correlated with increased stress resistance. The dwarf phenotype of chico homozygotes was also unnecessary for extension of life-span. The role of insulin/IGF signaling in regulating animal aging is therefore evolutionarily conserved.
\end{abstract}

Mutations that extend life-span illuminate the molecular mechanisms underlying aging and longevity. In Caenorhabditis elegans, mutation of the genes daf- 2 and age-1, which encode components of an insulin/IGF signaling (IIS) pathway, enhances stress resistance and increases adult life-span by up to $200 \%$ (1). This pathway also controls the formation of dauer larvae, which are developmentally arrested, stress resistant, long-lived, and produced in response to crowding and reduced food (2). Potentially, insulin/ IGF mutants could be long-lived by virtue of expression of dauer longevity in the adult, in which case the extension of adult life-span by these mutations could be a peculiarity of C. elegans. We examined whether the role of IIS in aging has been evolutionarily conserved and therefore might also operate in humans.

In the fruit fly Drosophila melanogaster, the insulin/IGF receptor INR, the insulin receptor substrate $\mathrm{CHICO}$, the phosphatidylinositol 3-kinase (PI3K) Dp110/ p60, and the PI3K target protein kinase B (PKB, also known as DAkt1) form a signaling pathway that regulates growth and size (3-7). We examined the effects on aging of hypomorphic mutations in Inr (equivalent to daf-2) and $P K B$, and null mutations in chico and the catalytic (Dp110, equivalent to age-1) and adapter ( p60) PI3K subunits (8). All mutants were tested as heterozygotes. We also tested chico ${ }^{1}$ (3) and $P K B^{3}$ (9) homozygotes and In $r^{G C 25} /$ In $r^{E 19}$ transheterozygotes, which form viable dwarf adults. The remaining mutations were homozygous lethal.

Most mutants tested had normal or significantly decreased life-span (10). For example, $P K B^{3}$ homozygotes and $I n r^{G C 25}$ / $I n r^{E 19}$ flies were short-lived. By contrast, chico $^{1}$ extended life-span (Figure 1). Homozygous chico ${ }^{1}$ females exhibited an increase of median and maximum life-span of up to 48 and $41 \%$, respectively. chico ${ }^{1}$ heterozygotes also exhibited increases in median life-span of up to 36 and $13 \%$ in females and males, respectively. Homozygous males, however, were slightly short-lived.

To confirm that chico ${ }^{1}$ itself extended lifespan, we tested the effect on life-span of pCSR4-chico, a P element containing chico $(+)$. This construct fully rescues the dwarf phenotype of chico ${ }^{1}(3)$. chico ${ }^{1}$ was crossed to two stocks containing independent pCSR4-chico insertions (pCSR4chico 1.1 and 2.3). As a control, chico ${ }^{1}$ was also crossed to the base stock in which the $\mathrm{P}$ element insertions were made. Progeny with either two copies (chico ${ }^{1}$ heterozygotes with one chico transgene) or one copy ( chico $^{1}$ heterozygotes alone) of chico(+) were compared (11). The rescue construct significantly reduced lifespan relative to the $+/$ chico $^{1}$ control. The median female life-span of 54 days in +/ chico ${ }^{1}$ was reduced to 46 days in $+/$ chico $^{1}$,
$+/ p$ CSR4-chico 1.1 flies and 52 days in +/ chico $^{1},+/ p$ CSR4-chico 2.3 flies $(P=0.0002$ and 0.0243 , respectively). Similar effects were observed in males (10). Thus, mutation of chico itself increases life-span. Because chico ${ }^{1}$ is a null allele, its effect on lifespan indicates that the wild-type chico gene acts to accelerate aging.

Of the mutations tested, only chico ${ }^{1}$ increased life-span. This may be because the effect of reduced IIS on life-span depends on the degree to which signaling is reduced. Unlike the other null mutations in IIS genes tested, chico ${ }^{1}$ is not homozygous lethal, presumably because the INR receptor can signal to PI3K directly, as well as indirectly via CHICO (3). Thus, chico $^{1}$ mutants may be long-lived because of the relatively mild reduction in pathway activity that they bring about. Notably, severe IIS mutations in C. elegans can cause premature mortality in some adults, although the maximum life-span of populations is invariably increased (1). This is probably why $\operatorname{In} r^{G C 25} / \operatorname{In} r^{E 19}$ flies are shortlived: Demographic analysis indicates that a reduction in the agespecific mortality rate acceleration occurs, whose effect on survival is masked by an elevated rate of age-independent mortality (12). Furthermore, a different heteroallelic Drosophila Inr mutant to that tested here exhibits an $85 \%$ increase in female life-span (13). By contrast, in shortlived $P K B^{3}$ populations, no reduction in mortality rate acceleration is seen (12). This raises the possibility that a second pathway downstream of chico might regulate aging in Drosophila. Interestingly, $\mathrm{CHICO}$ contains potential binding sites for the Drk/Grb2 docking protein, consistent with signaling via Ras/mitogenactivated protein kinase.

We next investigated whether extension of life-span by chico ${ }^{1}$ was mediated by processes previously shown to affect aging. A reduction in fecundity extends life-span in Drosophila females (14, 15); chico $^{1}$ heterozygous females have reduced fecundity, and the homozygotes are almost sterile $(3,12)$. To test whether the increased life-span of chico ${ }^{1}$ females was due to reduced fecundity, we examined the interactions between chico ${ }^{1}$ and the dominant, female-sterile mutant $o v o^{D 1}$. This mutation blocks oogenesis at stage 4 , before vitellogenesis commences (16), and extends female life-span (15). If chico $^{1}$ extends female life-span by the exact same mechanism as $o v o^{D 1}$, then the three sterile genotypes (chico ${ }^{1},+/$ ovo $^{D 1}$, and $+/ o v o^{D 1}+/$ chico $^{1}$ ) should have similar life-spans and live longer than the subfertile chico $^{1}$ heterozygotes. In fact, 
the chico homozygotes lived significantly longer than all other genotypes (Figure 2). In addition, the partially fertile chico $^{1}$ heterozygotes lived as long as the sterile flies that were heterozygous for both $o v o^{D 1}$ and chico $^{1}$ and lived significantly longer than the sterile ovo ${ }^{D 1}$ heterozygotes. The effect of chico ${ }^{1}$ on female lifespan is therefore not a consequence of the same mechanism of reduced fecundity as is produced by $o v o^{D 1}$. If $c h i c o^{1}$ does extend female life-span through an effect on reproductive effort, the interaction must occur through some process other than oogenesis (for instance yolk protein synthesis) or before stage 4 in oogenesis because $0 v o^{D 1}$ flies are blocked at that stage.

In C. elegans, long-lived IIS mutants are stress resistant and overexpress the antioxidant enzyme superoxide dismutase (SOD) (1). We examined the resistance of chico $^{1}$ flies to three stressors, but only one showed any correspondence with life-span (17) (Figure 3). No resistance to heat stress $\left(37^{\circ} \mathrm{C}\right)$ was seen (Figure 3A). Slight resistance to oxidative stress (methyl viologen) was observed in chico $^{1}$ heterozygotes but not in homozygotes (Figure 3B). However, some correspondence between starvation resistance and life-span was seen (Figure 3C). Increased SOD levels were seen in chico ${ }^{1}$ homozygotes but not in heterozygotes (17) (Figure 4). Thus, modulation by IIS of longevity, and of SOD levels, has evidently been conserved between C. elegans and Drosophila. Furthermore, effects of this pathway on fertility are widespread $(1,3,18)$. However, effects on stress resistance are not well conserved, nor do any of the above associated affects appear to be causal in extending life-span.

Our results raise the question of whether IIS regulates aging in mammals. Whereas both the C. elegans and Drosophila genomes contain a single insulin/IGF receptor, mammals possess distinct receptors for insulin and IGF-I, plus a third insulin receptor-like receptor of unknown function. Potentially, any or all of these receptors may play a role in regulating aging. Caloric restriction $(\mathrm{CR})$, which increases life-span in rodents (19), and possibly primates (20), reduces circulating levels of both insulin and IGF-I $(21,22)$. In the case of IGF$\mathrm{I}$, there is further evidence for a role in the control of longevity $(22,23)$. Growth hormone $(\mathrm{GH})$ acts via IGF-I to control mammalian body size, and circulating IGF-I levels correlate with body size in mice, dogs, and humans $(23,24)$. Furthermore, CR can reduce body size (22). In mice and dogs (and possibly humans), there is a marked negative correlation be- tween body size and longevity (24-26). In addition, long-lived Ames hypopituitary mouse dwarves are deficient in GH and other pituitary hormones and have reduced circulating IGF-I (27). Mutation of the human equivalent of the Ames dwarf gene, Prop-1, also causes dwarfism and, possibly, delayed aging (28). The Laron dwarf mouse, which has no GH receptor and very low IGF-I levels, exhibits lifespan increases of up to $55 \%(29)$.

Whereas the effects of $\mathrm{chico}^{1}$ on development that result in reduced body size are recessive, its effects on life-span are


Figure 1. Effect of chico ${ }^{1}$ on life-span. Median life-spans and sample sizes, respectively, for females are as follows. $(\mathbf{A})+/+, 53$ days and $N=119 ;+/$ chico $^{1}, 56$ days and $N=128$. (B) $+/+, 44$ days and $N=105 ;+/$ chico $^{1}, 60$ days and $N=100 ;$ chico $^{1}, 65$ days and $N=86$. $(\mathbf{C})+/+, 49$ days and $N=61$; chico ${ }^{1}, 65$ days and $N=67$. (D) $+/+, 46$ days and $N=172 ;+/$ chico $^{1}, 54$ days and $N=171$; chico $^{1}, 58$ days and $N=176$. Data for males are as follows. $(\mathbf{E})+/+, 54$ days and $N=$ 136; $+/$ chico $^{1}, 56$ days and $N$ 5135. (F) $+/+, 57$ days and $N=104 ;+/$ chico $^{1}, 62$ days and $N=$ 140. $(\mathbf{G})+/+, 59$ days and $N=54$; chico $^{1}, 50$ days and $N=48$. Statistical comparisons (log rank test) are as follows for females: $+/$ chico $^{1}$ versus $+/+, P<0.0001(\mathrm{~A}, \mathrm{~B}$, and $\mathrm{D})$; chico $^{1}$ versus + / chico $^{1}, P=0.0008$ and, 0.0001 (B and $\mathrm{D}$, respectively); and $c h i c 0^{1}$ versus $+/+, P<0.0001$ (B, $C$, and D). Statistical comparisons (log rank test) are as follows for males: $+/$ chico $^{1}$ versus $+/+$, $P<0.0001$ and $<0.0866$ (not significant) (E and $F$, respectively). In all panels, circles represent chico $^{1} /$ chico $^{1}$; squares represent $+/$ chico $^{1}$, and triangles represent $+/+$. 
Figure 2. Effects of chico $^{1}$ and ovo $^{D 1}$ on female lifespan. Median life-spans and sample sizes, respectively, are as follows: $+/+, 44$ days and $\mathrm{N}$ $=105 ;+/$ ovo $^{D 1}, 50$ days and $N=90$; +/ chico $^{1}, 60$ days and $N=100 ;+/$ chico $^{1}+$ / ovo $^{D 1}, 59$ days and $N=93$; and $\mathrm{chico}^{1}$, 65 days and $N=86$. Statistical compari-

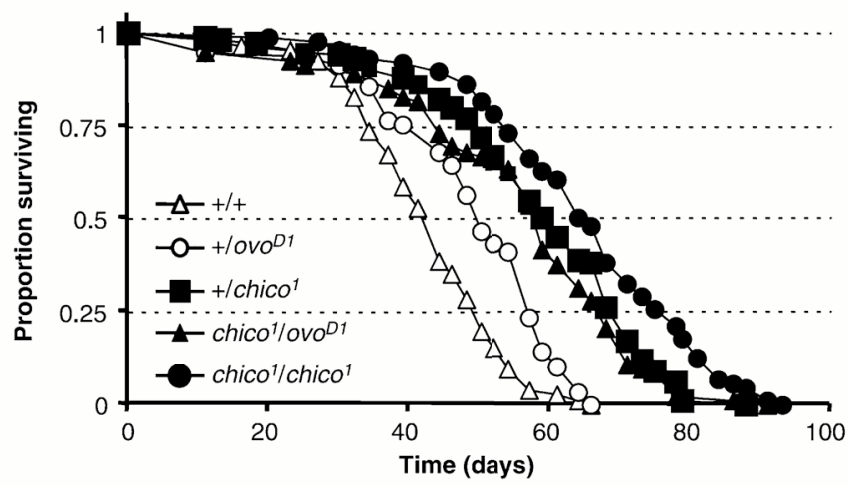

sons (log rank test) are as follows: +/+ versus +/ovo ${ }^{D 1}, P<0.0001$; +/ovo ${ }^{D 1}$ versus +/chico ${ }^{1}+$ ovo $^{D 1}$, or $+/$ chico $^{1}, P<0.0001 ;+/$ chico $^{1}+/$ ovo $^{D 1}$ versus $+/$ chico $^{1}$, no significant difference; and chico $^{1}$ versus $+/$ chico $^{1}+/$ ovo $^{D 1}$ or $+/$ chico $^{1}, P=0.0008$ in each case.

Figure 3. Effect of chico $^{1}$ on stress resistance. Histograms show mean survival time. Probability that values are identical to wild type is indicated as follows: *, $0.05>P>0.01 ; * *$, $P<0.01$ (log rank). (A) Heat stress resistance. Sample sizes for females were as follows: 49 $(+/+), 50\left(+/\right.$ chico $\left.^{1}\right)$, and 51 (chico $\left.{ }^{1}\right)$ on day 1 and $49(+/+)$, $50\left(+/\right.$ chico $\left.^{1}\right)$, and $52\left(\right.$ chico $\left.^{1}\right)$ on day 2. Sample sizes for males were as follows: $50(+/+), 50$ $\left(+/\right.$ chico $\left.^{1}\right)$, and 50 $\left(\right.$ chico $\left.^{1}\right)$ on day 1 and $52(+/+), 51$ $\left(+/\right.$ chico $\left.^{1}\right)$, and 47 $\left(\right.$ chico $\left.^{1}\right)$ on day 2. (B)
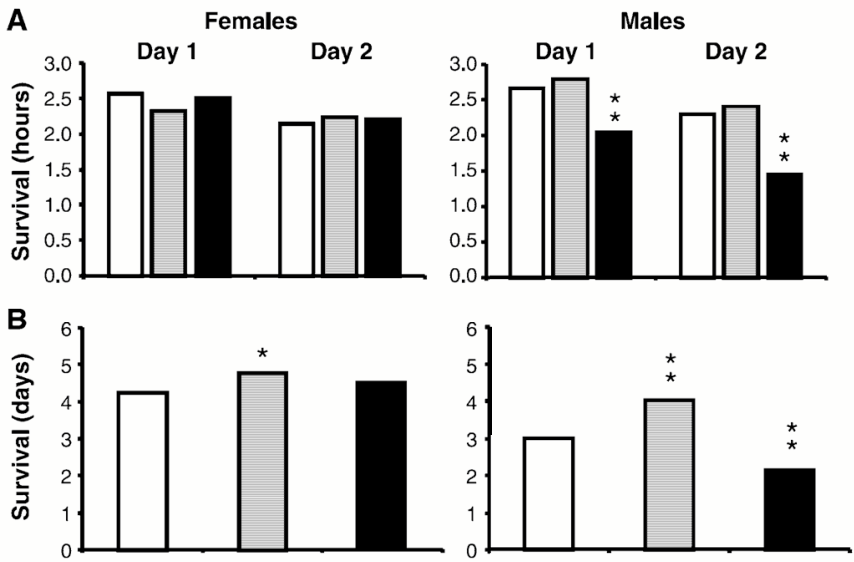

\section{C}

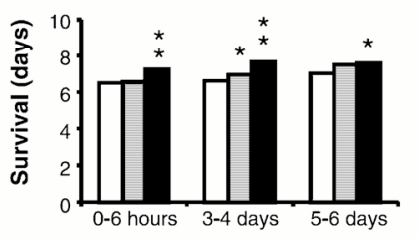

Age at starvation

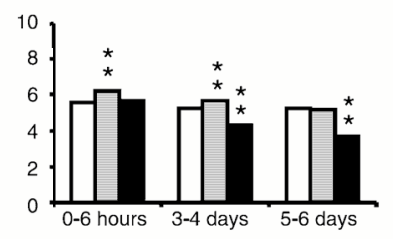

Age at starvation
Oxidative stress (methyl viologen) resistance. Sample sizes for females were $100(+/+), 100(+/$ chico $\left.^{1}\right)$, and $99\left(\right.$ chico $\left.^{1}\right)$. Sample sizes for males were $99(+/+), 100\left(+/\right.$ chico $\left.^{1}\right)$, and $69\left(\right.$ chico $\left.^{1}\right)$. (C) Starvation resistance. Sample sizes for females were as follows: from 0 to 6 hours, $150(+/+)$, $150\left(+/\right.$ chico $\left.^{1}\right)$, and $150\left(\right.$ chico $\left.^{1}\right)$; from 3 to 4 days, $147(+/+), 148\left(+/\right.$ chico $\left.^{1}\right)$, and $146\left(\right.$ chico $\left.^{1}\right)$; and from 5 to 6 days, $150(+/+), 150\left(+/\right.$ chico $\left.^{1}\right)$, and $151\left(\right.$ chico $\left.^{1}\right)$. Sample sizes for males were as follows: from 0 to 6 hours, $150(+/+), 150\left(+/\right.$ chico $\left.^{1}\right)$, and $101\left(\right.$ chico $\left.^{1}\right)$; from 3 to 4 days, 149 $(+/+), 148\left(+/\right.$ chico $\left.^{1}\right)$, and $101\left(\right.$ chico $\left.^{1}\right)$; and from 5 to 6 days, $149(+/+), 149\left(+/\right.$ chico $\left.^{1}\right)$, and 97 $\left(\right.$ chico $\left.^{1}\right)$. In all panels, white bars represent $+/+$, gray bars represent $+/$ chico $^{1}$, and black bars represent $\mathrm{chico}^{1} /$ chico $^{1}$.

Figure 4. Effect of chico ${ }^{1}$ on total superoxide dismutase (Cu/Zn and Mn SOD) activity. Histograms show mean values with $95 \%$ confidence intervals. Probability that the values are identical to that of the wild type is in-

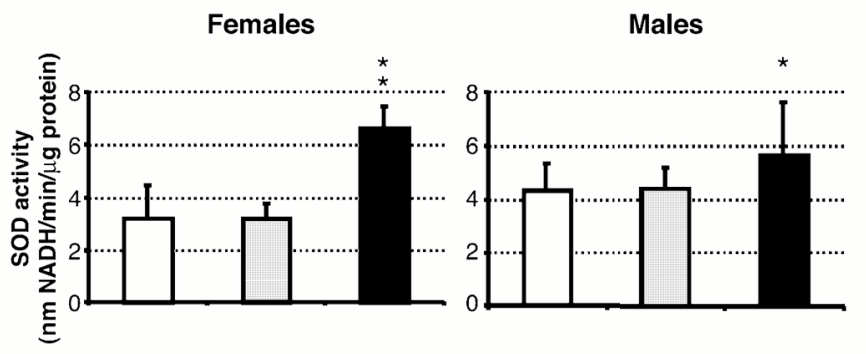

dicated as follows: *, $0.05>P>0.01 ; * *, P<0.01$ (paired $t$ test). Replicate assays were conducted at several different times. Sample sizes for females were $6(+/+), 6\left(+/\right.$ chico $\left.^{1}\right)$, and 4 $\left(\right.$ chico $\left.^{1}\right)$. Sample sizes for males were $5(+/+), 6\left(+/\right.$ chico $\left.^{1}\right)$, and $4\left(\right.$ chico $\left.^{1}\right)$. White bars represent $+/+$, gray bars represent $+/$ chico $^{1}$, and black bars represent $\mathrm{chico}^{1} / \mathrm{chico}^{1}$.
Together, our results with fruit flies and recent findings with nematodes and mice suggest that the role of IIS (perhaps IGF-I in mammals) in regulating longevity is evolutionarily conserved throughout the animal kingdom.

\section{References and Notes}

1. Guarente, C. Kenyon, Nature 408, 255 (2000). 2. D. L. Riddle, P. S. Albert, in C. elegans II, D. L. Riddle et al., eds. (Cold Spring Harbor Press, Plainview, NY, 1997), pp. 739-768.

3. R. Böhni et al., Cell 97, 865 (1999).

4. C. Chen, J. Jack, R. S. Garofalo, Endocrinology 137, 846 (1996).

5. S. J. Leevers, D. Weinkove, L. K. MacDougall, M. D. WaterPeld, EMBO J. 15, 6584 (1996).

6. J. Verdu, M. A. Buratovich, E. L. Wilder, M. J. Birnbaum, Nature Cell Biol. 1, 500 (1999).

7. D. Weinkove, S. J. Leevers, Curr. Opin. Genet. Dev. 10, $75(2000)$

8. Five Inr mutations were examined: $I n r[P Z]$ and $I n r^{304}$ [R. Fernandez et al., EMBO J. 14, 3373 (1995)] and InrGC25, In EC34, and InrE19 (4). Also, one Dp110 null $\left(D p 110^{A}\right)(5)$, one $p 60$ null $\left(p 60^{A}\right)$ [D. Weinkove, S. J. Leevers, L. K. MacDougall, M. D. WaterPeld, J. Biol. Chem. 272, 14606 (1997)], one chico null $\left(\right.$ chico $\left.^{1}\right)(3)$, and one $P K B$ hypomorph $\left(P K B^{3}\right)$ (provided to E.H. by C. Zucker) were examined. All mutations in this study were backcrossed four times into the outbred, wildtype Dahomey genetic background before life-span and other traits were measured. For further details, see supplemental information (12).

9. H. Stocker, E. Hafen, unpublished results.

10. D. J. Clancy et al., data not shown.

11. pCSR4-chico 1.1 and 2.3 (insertions on the third chromosome) were generated in a base stock marked with yellow, white $(y w)$. Females of the chico ${ }^{1}$ Dahomey stock were crossed either to males from each pCSR4chico stock or to males from the yw base stock.

12. Supplemental information is available at http://www.sciencemag.org/cgi/content/ article.

13. M. Tatar et al., Science 292, 107 (2001).

14. J. Maynard Smith, J. Exp. Biol. 35, 832 (1958).

15. C. M. Sgrò, L. Partridge, Science 286, 2521 (1999).

16. B. Oliver, N. Perrimon, A. P. Mahowald, Genes Dev. 1, 913 (1987)

17. Oxidative stress resistance was measured with methyl viologen (Paraquat). SOD was measured by the oxidation of the reduced form of nicotinamide adenine dinucleotide. For details of these measurements and heat stress and starvation tests, see supplemental information (12).

18. D. J. Burks et al., Nature 407, 377 (2000).

19. C. McCay, M. Crowell, L. Maynard, J. Nutr. 10, 63 (1935).

20. M. A. Lane et al., Proc. Natl. Acad. Sci. U.S.A. 93 , 4159 (1996).

21. R. S. Sohal, R. Weindruch, Science 273, 59 (1996).

22. W. E. Sonntag et al., J. Gerontol. 54A, B521 (1999).

23. A. Bartke, in The Molecular Genetics of Aging, S Hekimi, ed., vol. 29 of Results and Problems in Cell Differentiation (Springer-Verlag, Berlin, 2000), pp. 181-202.

24. J. Eigenmann, D. Patterson, E. R. Froesch, Acta Endocrinol. 106, 448 (1984).

25. R. Miller, C. Chrisp, W. Atchley, J. Gerontol. 55A B455 (2000).

26. T. Samaras, L. Storms, Bull. WHO 70, 259 (1992).

27. H. M. Brown-Borg, K. E. Borg, C. J. Meliska, A. Bartke, Nature 384, 33 (1996).

28. C. Krzisnik et al., J. Endocr. Genet. 1, 9 (1999).

29. K. Coschigano, D. Clemmons, L. Bellush, J. Kopchick, Endocrinology 141, 2608 (2000).

30 . This work was supported by the Biotechnology and Biological Sciences Research Council Science of Ageing initiative (grant 31/SAG09982), the National Environment Research Council, the Royal Society, the Ludwig Institute for Cancer Research, and the National Institute on Aging (grant AG08761). We thank $\mathrm{S}$. Pletcher for assistance with biodemographic analysis and two anonymous reviewers for improvements to the paper. 


\section{Supplementary Material}

\section{Methods}

\section{Fly culture and handling}

All flies were reared at standard low density, and virgins were collected and handled with $\mathrm{CO}_{2}$ anesthesia. Adults of the same sex were kept at a density of 10 per vial. Flies were transferred to fresh medium, and deaths were scored three times per week. The survival of all genotypes was compared with the base stock by using log rank tests [R. G. Miller, Survival Analysis (Wiley, New York, 1981)].

The Dahomey stock used to outcross the mutations examined has been in laboratory culture for 30 years in population cages. Stocks cultured in these conditions retain adult survival rates comparable to those of fly stocks freshly collected from nature [C. Sgrò, L. Partridge, Am. Nat. 156, 341 (2000)]. Any positive effects of the mutations on adult survival could therefore not be attributed to rescue of lowered life-span caused by inbreeding or laboratory adaptation.

\section{Biodemographic analysis}

In order to investigate whether differences in life-span between IIS mutants resulted from increased initial (non-age-dependent) mortality or from changes in the rate of acceleration of mortality with increasing age, we analyzed patterns of age-specific mortality in these strains. The best fit to changes in age-specific mortality was with the Gompertz-Makeham model $\left[m(t)=A_{0} e^{G t}+M_{0}\right]$, which characterizes mortality profiles $[m(t)$, mortality rate at time $t]$ in terms of three variables: (i) an initial mortality rate (IMR, or $A_{0}$ ), (ii) a mortality rate acceleration constant (the Gompertz coefficient, $G$ ), and (iii) the Makeham parameter $\left(M_{0}\right)$, which is an age-independent mortality constant, distinct from $A_{0}$, which may reflect environmental effects. An increase in the IMR would indicate that the strain was merely sick, whereas an increase or decrease in the Gompertz coefficient is consistent with accelerated or retarded aging, respectively. Our most sensitive test, containing the most scoring intervals, is that shown in Fig. 1B of the report. Here, the maximum likelihood estimate of $G$ was reduced from 0.2011 in controls (Dahomey) to 0.1455 in chico $^{1}$ homozygotes ( $P=0.0493$; $\chi^{2}$ value, derived from the difference between likelihood values of parameterconstrained and unconstrained models). Thus, chico $^{1}$ reduces the rate of aging. By contrast, the estimates of $A_{0}$ did not differ significantly between controls and chico ${ }^{1}$ mutants, nor did either parameter differ from controls in chico $^{1}$ heterozygotes. In another trial, the parameter estimate in Inr transheterozygote females for $G$ was reduced $(P=0.0091)$, whereas that for $A_{0}$ was increased $(P=0.0080)$ relative to controls. This suggests that these flies age more slowly but are also frailer than the wild type (see Web table 1 ).

Supplemental Table 1. Maximum likelihood estimate of Gompertz-Makeham parameters.

\begin{tabular}{|c|c|c|c|}
\hline Line & $A 0_{0}$ & $G$ & $M 0_{0}$ \\
\hline Wild type & 0.00115 & 0.18802 & $2.06 \mathrm{e}-9^{-9}$ \\
\hline InrE19 $9^{E 19} /$ InrGC25 $5^{G C 25}$ & 0.02158 & 0.08768 & $2.06 \mathrm{e}-9^{-9}$ \\
\hline$P K B 3^{3}$ & 0.00486 & 0.15060 & $2.06 \mathrm{e}-9^{-9}$ \\
\hline
\end{tabular}


This agrees with the results of Tatar et al. [see (13) in report]. In $P K B^{3}$ homozygotes, parameter estimates did not differ from controls, although an increased $A_{0}$ was borderline significant $(P=0.0664)$.

\section{Measurement of female fecundity}

Flies were reared at constant low density in bottles, collected as virgins, and placed individually in vials with food medium and live yeast paste. In one experiment, egg production between days 4 and 14 was monitored; in the second experiment, eggs between days 4 and 16 were counted. In both experiments, chico $^{1}$ heterozygotes laid significantly fewer eggs than did the controls (first experiment mean $=231$ versus mean $=305, P<0.0001,+/+, N=49 ;+$ chico $^{1}, N=57$; second experiment mean $=469$ versus mean $=566, P=0.0152,+/+, N=33 ;+/$ chico $^{1}, N=35$ ).

\section{Measurement of resistance to heat stress, oxidative stress, and starvation}

For stress resistance experiments, mortality was monitored, mean survival times were calculated, and statistical analysis was performed by the nonparametric log rank test, unless otherwise stated. To measure resistance to heat stress, we placed 5- to 7-day-old virgins in a vial in single-sex groups (10 to a vial, with moistened filter paper and a cotton wool plug with an air-permeable plastic cap), then incubated them at $37^{\circ} \mathrm{C}$ in a water bath. Time to immobility was scored with independent groups of flies on two consecutive days. To measure resistance to oxidative stress, we put 3- to 4-day-old virgins in a vial (9 or 10 flies to a vial), with eight filter paper discs soaked with $500 \mu \mathrm{l}$ of $5 \%$ sucrose and $20 \mathrm{mM}$ methyl viologen (Paraquat). To measure starvation resistance, we placed 0-, 3-, and 5-day-old virgins in single-sex groups in vials with $1 \%$ agar and $0.3 \%$ methyl 4-hydroxybenzoate (Nipagin).

\section{Superoxide dismutase (SOD) assays}

To assay total SOD activity, we homogenized groups of 4-day-old virgins in $500 \mu \mathrm{l}$ of $50 \mathrm{mM}$ tris (pH 8.0 ) and $0.125 \mathrm{mM} \mathrm{NaCl}$, then centrifuged them at $2000 \mathrm{~g}$ for $5 \mathrm{~min}$. The supernatant was centrifuged in a size cutoff microconcentrator (10,000 dalton, Centricon-10, Millipore Corporation) to remove peptides and small molecules with SOD-like activity. The protein retentate was diluted 30-fold in homogenizing buffer, then reconcentrated in the same manner. Soluble protein concentration was assayed with the bicinchoninic acid (BCA) protein assay (Pierce Company). Total SOD activity was measured by using the method of F. Paoletti and A. Mocali [Methods Enzymol. 186, 209 (1990)]. The oxidation of NADH depends on the availability of superoxide radicals in solution. NADH oxidation was monitored by decreasing $A_{340 \mathrm{~nm}}$. For a control, NADH oxidation was monitored for 20 min under superoxide-producing conditions. Statistical analysis was by paired $t$ tests, pairing representing assays that were done on the same day.

If you have any questions, please contact David Gems (d.gems@galton.ucl.ac.uk) or Linda Partridge (l.partridge@ucl.ac.uk). 